\title{
Interpolation of Weighted Extremal Functions
}

\section{Alexander Rashkovskii ${ }^{1}$ if}

Received: 7 June 2019 / Revised: 18 January 2021 / Accepted: 22 February 2021 /

Published online: 16 March 2021

(C) The Author(s) 2021

\begin{abstract}
An approach to interpolation of compact subsets of $\mathbb{C}^{n}$, including Brunn-Minkowski type inequalities for the capacities of the interpolating sets, was developed in [8] by means of plurisubharmonic geodesics between relative extremal functions of the given sets. Here we show that a much better control can be achieved by means of the geodesics between weighted relative extremal functions. In particular, we establish convexity properties of the capacities that are stronger than those given by the BrunnMinkowski inequalities.
\end{abstract}

Keywords Plurisubharmonic geodesic $\cdot$ Coconvex sets · Brunn-Minkowski inequality $\cdot$ Relative extremal function

Mathematics Subject Classification 32U15 $\cdot 32 \mathrm{U} 20 \cdot 52 \mathrm{~A} 20 \cdot 52 \mathrm{~A} 40$

\section{Introduction}

Classical complex interpolation of Banach spaces, due to Calderón [5] (see [3] and, for more recent developments, [7]) is based on constructing holomorphic hulls generated by certain families of holomorphic mappings. A slightly different approach proposed in [8] rests on plurisubharmonic geodesics. The notion has been originally considered, starting from 1987, for metrics on compact Kähler manifolds (see [10] and the bibliography therein), while its local counterpart for plurisubharmonic functions on bounded hyperconvex domains of $\mathbb{C}^{n}$ was introduced more recently in [4] and [18], see also [1].

In the simplest case, the geodesics we need can be described as follows. Denote by $A=\{\zeta \in \mathbb{C}: 0<\log |\zeta|<1\}$ the annulus bounded by the circles $A_{j}=\{\zeta$ : $\log |\zeta|=j\}, j=0,1$. Let $\Omega$ be a bounded hyperconvex domain in $\mathbb{C}^{n}$. Given two plurisubharmonic functions $u_{0}, u_{1}$ in $\Omega$, equal to zero on $\partial \Omega$, we consider the class

\footnotetext{
$凶$ Alexander Rashkovskii

alexander.rashkovskii@uis.no

1 Tek/Nat, University of Stavanger, 4036 Stavanger, Norway
} 
$W$ of all plurisubharmonic functions $u(z, \zeta)$ in $\Omega \times A$, such that

$$
\limsup _{\zeta \rightarrow A_{j}} u(z, \zeta) \leq u_{j}(z) \quad \forall z \in \Omega
$$

Its Perron envelope $\mathcal{P}_{W}(z, \zeta)=\sup \{u(z, \zeta): u \in W\}$ belongs to the class and satisfies $\mathcal{P}_{W}(z, \zeta)=\mathcal{P}_{W}(z,|\zeta|)$, which gives rise to the functions

$$
u_{t}(z):=\mathcal{P}_{W}\left(z, e^{t}\right), \quad 0<t<1,
$$

the geodesic between $u_{0}$ and $u_{1}$. When the functions $u_{j}$ are bounded, the geodesic $u_{t}$ tends to $u_{j}$ as $t \rightarrow j$, uniformly on $\Omega$. One of the main properties of the geodesics is that they linearize the pluripotential energy functional

$$
\mathcal{E}(u)=\int_{\Omega} u\left(d d^{c} u\right)^{n}
$$

which means

$$
\mathcal{E}\left(u_{t}\right)=(1-t) \mathcal{E}\left(u_{0}\right)+t \mathcal{E}\left(u_{1}\right)
$$

see the details in [4] and [18].

In [18], this was adapted to the case when the endpoints $u_{j}$ are relative extremal functions $\omega_{K_{j}}$ of non-pluripolar compact sets $K_{0}, K_{1} \subset \Omega$; we recall that

$$
\omega_{K}(z)=\omega_{K, \Omega}(z)=\limsup _{y \rightarrow z} \mathcal{P}_{\mathcal{N}_{K}}(y)
$$

where $\mathcal{N}_{K}$ is the collection of all negative plurisubharmonic functions $u$ in $\Omega$ with $\left.u\right|_{K} \leq-1$, see [14]. Note that

$$
\mathcal{E}\left(\omega_{K}\right)=-\operatorname{Cap}(K)
$$

where

$$
\operatorname{Cap}(K)=\operatorname{Cap}(K, \Omega)=\left(d d^{c} \omega_{K}\right)^{n}(\Omega)=\left(d d^{c} \omega_{K}\right)^{n}(K)
$$

is the Monge-Ampère capacity of $K$ relative to $\Omega$.

If each $K_{j}$ is polynomially convex (i.e., coincides with its polynomial hull), then the functions $u_{j}=-1$ exactly on $K_{j}$ are continuous on $\bar{\Omega}$, and the geodesics $u_{t} \in$ $C(\bar{\Omega} \times[0,1])$. Let

$$
K_{t}=\left\{z \in \Omega: u_{t}(z)=-1\right\}, \quad 0<t<1
$$

then (1) implies:

$$
\operatorname{Cap}\left(K_{t}\right) \leq(1-t) \operatorname{Cap}\left(K_{0}\right)+t \operatorname{Cap}\left(K_{1}\right) .
$$

As was shown in [19], the functions $u_{t}$ in general are different from the relative extremal functions of $K_{t}$. Moreover, if the sets $K_{j}$ are Reinhardt (toric), then $u_{t}=\omega_{K_{t}}$ 
for some $t \in(0,1)$ only if $K_{0}=K_{1}$, so an equality in (3) is never possible unless the geodesic degenerates to a point.

Furthermore, in the toric case, the capacities (with respect to the unit polydisk $\mathbb{D}^{n}$ ) were proved in [8] to be not just convex functions of $t$, as is depicted in (3), but logarithmically convex:

$$
\operatorname{Cap}\left(K_{t}, \mathbb{D}^{n}\right) \leq \operatorname{Cap}\left(K_{0}, \mathbb{D}^{n}\right)^{1-t} \operatorname{Cap}\left(K_{1}, \mathbb{D}^{n}\right)^{t}
$$

This was done by representing the capacities, due to [2], as (co)volumes of certain sets in $\mathbb{R}^{n}$ and applying convex geometry methods to an operation of copolar addition introduced in [19]. Furthermore, the sets $K_{t}$ in the toric situation were shown to be the geometric means (multiplicative combinations) of $K_{j}$, exactly as in the Calderón complex interpolation theory. And again, an equality in (4) is possible only if $K_{0}=K_{1}$. It is worth mentioning that the volumes of $K_{t}$ satisfy the opposite Brunn-Minkowski inequality [6]:

$$
\operatorname{Vol}\left(K_{t}\right) \geq \operatorname{Vol}\left(K_{0}\right)^{1-t} \operatorname{Vol}\left(K_{1}\right)^{t}
$$

In this note, we apply the geodesic technique to weighted relative extremal functions

$$
u_{j}^{c}=c_{j} \omega_{K_{j}}, \quad c_{j}>0
$$

the sets $K_{t}$ being replaced with the sets $K_{t}^{c}$ where the functions $u_{t}^{c}$ attain their minimal values, $-c_{t}$. The function $t \mapsto c_{t}$ turns out to be convex; moreover, it is actually linear, $c_{t}=(1-t) c_{0}+t c_{1}$, provided $K_{0} \cap K_{1} \neq \emptyset$. With such an interpolation, one can have $u_{t}^{c}=c_{t} \omega_{K_{t}^{c}, \Omega}$ for a non-degenerate geodesic, in which case there is no loss in the transition from the functional $\mathcal{E}\left(u_{t}^{c}\right)$ to the capacity Cap $\left(K_{t}^{c}\right)$. And in any case, we establish the weighted inequality

$$
c_{t}^{n+1} \operatorname{Cap}\left(K_{t}^{c}\right) \leq(1-t) c_{0}^{n+1} \operatorname{Cap}\left(K_{0}\right)+t c_{1}^{n+1} \operatorname{Cap}\left(K_{1}\right),
$$

which, for a smart choice of the constants $c_{j}$, is stronger than (3) and even (in the toric case) than (4). In particular, it implies that the function

$$
t \mapsto\left(\operatorname{Cap}\left(K_{t}^{c}\right)\right)^{-\frac{1}{n+1}}
$$

is concave.

In the toric setting of Reinhardt sets $K_{j}$ in the unit polydisk, we show that the interpolating sets $K_{t}^{c}$ actually are the geometric means, so they do not depend on the weights $c_{j}$ and coincide with the sets $K_{t}$ in the non-weighted interpolation; we do not know if the latter is true in the general, non-toric setting.

Finally, we transfer the above results on the capacities of sets in $\mathbb{C}^{n}$ to the realm of convex geometry, developing thus the Brunn-Minkowski theory for volumes of (co)convex sets in $\mathbb{R}^{n}[8,12,19]$. 


\section{General Setting}

Here, we consider the general case of $u_{j}^{c}=c_{j} \omega_{K_{j}}$ with $c_{j}>0$ and $K_{j}$ non-pluripolar, compact, polynomially convex subsets of a bounded hyperconvex domain $\Omega$ of $\mathbb{C}^{n}$. In this situation, the functions $u_{j}^{c}(z)=-c_{j}$ precisely on $K_{j}$ and are continuous on $\bar{\Omega}$, the geodesics $u_{t}$ converge to $u_{j}$, uniformly on $\Omega$, as $t \rightarrow j$, and belong to $C(\bar{\Omega} \times[0,1])$, as in the non-weighted case dealt with in [18] and [8].

Denote:

$$
c_{t}=-\min \left\{u_{t}^{c}(z): z \in \Omega\right\}
$$

and

$$
K_{t}^{c}=\left\{z \in \Omega: u_{t}^{c}(z)=-c_{t}\right\}, \quad 0<t<1,
$$

the set where $u_{t}^{c}$ attains its minimal value on $\Omega$.

Theorem 1 In the above setting, we have:

(i) $c_{t} \leq(1-t) c_{0}+t c_{1}$, with an equality if $K_{0} \cap K_{1} \neq \emptyset$;

(ii) the function $t \mapsto c_{t}^{n+1} \mathrm{Cap}\left(K_{t}\right)$ is convex:

$$
c_{t}^{n+1} \operatorname{Cap}\left(K_{t}^{c}\right) \leq(1-t) c_{0}^{n+1} \operatorname{Cap}\left(K_{0}\right)+t c_{1}^{n+1} \operatorname{Cap}\left(K_{1}\right) ;
$$

(iii) if the weights $c_{j}$ are chosen such that

$$
c_{0}^{n+1} \operatorname{Cap}\left(K_{0}\right)=c_{1}^{n+1} \operatorname{Cap}\left(K_{1}\right),
$$

then the function

$$
V(t):=\left(\operatorname{Cap}\left(K_{t}^{c}\right)\right)^{-\frac{1}{n+1}}
$$

is concave and, consequently, the function

$$
\rho(t):=V(t)^{-1}=\left(\operatorname{Cap}\left(K_{t}^{c}\right)\right)^{\frac{1}{n+1}}
$$

is convex.

Proof (i) Consider $v_{j}=c_{j} \omega_{K}$ for $j=0,1$, where $K=K_{0} \cup K_{1}$. The set $K$ might be not polynomially convex, but $\omega_{K}$ is nevertheless a bounded plurisubharmonic function on $\Omega$ with zero boundary values, so the geodesic $v_{t}^{c}$ is well defined and converge to $v_{j}$, uniformly on $\Omega$, as $t \rightarrow j$ [18, Prop. 3.1]. Since $v_{j} \leq$ $u_{j}^{c}$, we have $v_{t}^{c} \leq u_{t}^{c}$. Assume $c_{0} \geq c_{1}$, then the corresponding geodesic $v_{t}^{c}=$ $\max \left\{c_{0} \omega_{K},-\left((1-t) c_{0}+t c_{1}\right)\right\}$, because the right-hand side is maximal in $\Omega \times A$ and has the prescribed boundary values at $t=0$ and $t=1$. Therefore:

$$
-c_{t} \geq \min \left\{v_{t}^{c}(z): z \in \Omega\right\} \geq-\left((1-t) c_{0}+t c_{1}\right)
$$


which proves the convexity of $c_{t}$.

To finish the proof of (i), let $z^{*} \in K_{0} \cap K_{1} \neq \emptyset$, then $-c_{t} \leq u_{t}^{c}\left(z^{*}\right)$. Since the convexity of the function $u_{t}^{c}\left(z^{*}\right)$ in $t$ implies $u_{t}^{c}\left(z^{*}\right) \leq-\left((1-t) c_{0}+t c_{1}\right)$, we get $c_{t} \geq(1-t) c_{0}+t c_{1}$ and thus the linearity.

(ii) Since $u_{j}^{c}=c_{j} \omega_{K_{j}}$, we have:

$$
\mathcal{E}\left(u_{j}\right)=c_{j}^{n+1} \int_{\Omega}\left(d d^{c} \omega_{K_{j}}\right)^{n}=-c_{j}^{n+1} \operatorname{Cap}\left(K_{j}\right), \quad j=1,2 .
$$

For any fixed $t$, the function $u_{t}^{c}=-c_{t}$ on $K_{t}^{c}$, so $u_{t}^{c} \leq-c_{t} \omega_{K_{t}^{c}}$. By [18, Cor. 2.2] this implies

$$
\mathcal{E}\left(u_{t}^{c}\right) \leq \mathcal{E}\left(c_{t} \omega_{K_{t}^{c}}\right)=-c_{t}^{n+1} \operatorname{Cap}\left(K_{t}^{c}\right),
$$

and (6) follows from the geodesic linearization property (1).

(iii) It suffices to prove the concavity of the function $V$. When the weights $c_{j}$ satisfy (7), inequality (6) rewrites as

$$
V(t) \geq \frac{c_{t}}{c_{0}} V(0)
$$

and, since

$$
c_{1}=\frac{V(1)}{V(0)} c_{0},
$$

this gives us

$$
V(t) \geq(1-t) V(0)+t V(1)
$$

which completes the proof.

The convexity/concavity results in this theorem are stronger than inequality (3) obtained in [18] by the geodesic interpolation $u_{t}$ of non-weighted extremal functions. In addition, the non-weighted geodesic $u_{t}$ is very unlikely to be the extremal function of the set $K_{t}$ (as shown in [19], this is never the case in the toric situation, unless $K_{0}=K_{1}$ ), while this is perfectly possible in the weighted interpolation. For example, given $K_{0} \Subset \Omega$, let

$$
K_{1}=\left\{z \in \Omega: \omega_{K_{0}}(z) \leq-\frac{1}{2}\right\},
$$

then $\omega_{K_{1}, \Omega}=\max \left\{2 \omega_{K_{0}, \Omega},-1\right\}$. For $c_{0}=2$ and $c_{1}=1$, we get:

$$
u_{t}^{c}=\max \left\{2 \omega_{K_{0}},-2+t\right\}=(2-t) \omega_{K_{t}^{c}}
$$


with

$$
K_{t}^{c}=\left\{z \in \Omega: \omega_{K_{0}}(z) \leq-1+t / 2\right\},
$$

so

$$
\operatorname{Cap}\left(K_{t}^{c}\right)=\left(1-\frac{t}{2}\right)^{-1}\left|\mathcal{E}\left(u_{t}^{c}\right)\right|=\left(1-\frac{t}{2}\right)^{-1-n} \operatorname{Cap}\left(K_{0}\right) .
$$

\section{Toric Case}

In this section, we assume $\Omega=\mathbb{D}^{n}$, the unit polydisk, and $K_{0}, K_{1} \subset \mathbb{D}^{n}$ to be non-pluripolar, polynomially convex compact Reinhardt (multicircled, toric) sets. Polynomial convexity of such a set $K$ means that its logarithmic image

$$
\log K=\left\{s \in \mathbb{R}_{-}^{n}:\left(e^{s_{1}}, \ldots, e^{s_{n}}\right) \in K\right\}
$$

is a complete convex subset of $\mathbb{R}_{-}^{n}$, i.e., $\log K+\mathbb{R}_{-}^{n} \subset \log K$; we will also say that $K$ is complete logarithmically convex. The functions $c_{j} \omega_{K_{j}}$ are toric, and so is their geodesic $u_{t}^{c}$. Note that since $K_{0} \cap K_{1} \neq \emptyset$, inequality (6) and the concavity/convexity statements of Theorem 1(iii) hold true.

It was shown in [8] that the sets $K_{t}$ defined by (2) for the geodesic interpolation of non-weighted toric extremal functions $\omega_{K_{j}}$ are, as in the classical interpolation theory, the geometric means $K_{t}^{\times}$of $K_{j}$. Here, we extend the result to the weighted interpolation, which shows, in particular, that the sets $K_{t}^{c}$ do not depend on the weights $c_{j}$. The relation $K_{t}^{\times} \subset K_{t}^{c}$ is easy, while the reverse inclusion is more elaborate; we mimic the proof of the corresponding relation for the non-weighted case [8] that rests on a machinery developed in [19].

Any toric plurisubharmonic function $u(z)$ in $\mathbb{D}^{n}$ gives rise to a convex function

$$
\check{u}(s)=u\left(e^{s_{1}}, \ldots, e^{s_{n}}\right), \quad s \in \mathbb{R}_{-}^{n},
$$

and the geodesic $u_{t}^{c}$ generates the function $\check{u}_{t}^{c}$, convex in $(s, t) \in \mathbb{R}_{-}^{n} \times(0,1)$.

Given a convex function $f$ on $\mathbb{R}_{-}^{n}$, we extend it to the whole $\mathbb{R}^{n}$ as a lower semicontinuous convex function on $\mathbb{R}^{n}$, equal to $+\infty$ on $\mathbb{R}^{n} \backslash \overline{\mathbb{R}_{-}^{n}}$, and we denote $\mathcal{L}[f]$ its Legendre transform:

$$
\mathcal{L}[f](x)=\sup _{y \in \mathbb{R}^{n}}\{\langle x, y\rangle-f(y)\}
$$

Evidently, $\mathcal{L}[f](x)=+\infty$ if $x \notin \overline{\mathbb{R}_{+}^{n}}$, and the Legendre transform is an involutive duality between convex functions on $\mathbb{R}_{+}^{n}$ and $\mathbb{R}_{-}^{n}$.

It was shown in [19] that for the relative extremal function $\omega_{K}=\omega_{K, \mathbb{D}^{n}}$

$$
\mathcal{L}\left[\check{\omega}_{K}\right]=\max \left\{h_{Q}+1,0\right\},
$$


where

$$
h_{Q}(a)=\sup _{s \in Q}\langle a, s\rangle, \quad a \in \mathbb{R}_{+}^{n}
$$

is the support function of the convex set $Q=\log K \subset \mathbb{R}_{-}^{n}$. Therefore, for a weighted relative extremal function $u=c \omega_{K}$, we have:

$$
\mathcal{L}[\check{u}](a)=c_{j} \mathcal{L}\left[\check{\omega}_{K_{j}}\right]\left(c_{j}^{-1} a\right)=\max \left\{h_{Q}(a)+c_{j}, 0\right\}
$$

Theorem 2 Given two non-pluripolar complete logarithmically convex compact Reinhardt sets $K_{0}, K_{1} \subset \mathbb{D}^{n}$ and two positive numbers $c_{0}$ and $c_{1}$, let $u_{t}^{c}$ be the geodesic connecting the functions $u_{0}=c_{0} \omega_{K_{0}}$ and $u_{1}=c_{1} \omega_{K_{1}}$. Then the interpolating sets $K_{t}^{c}$ defined by (5) coincide with the geometric means:

$$
K_{t}^{\times}:=K_{0}^{1-t} K_{1}^{t}=\left\{z:\left|z_{l}\right|=\left|\eta_{l}\right|^{1-t}\left|\xi_{l}\right|^{t}, 1 \leq l \leq n, \eta \in K_{0}, \xi \in K_{1}\right\} .
$$

Proof Since the sets $K_{t}^{\times}$and $K_{t}^{c}$ are complete logarithmically convex, it suffices to prove that $Q_{t}:=\log K_{t}^{\times}$coincides with $Q_{t}^{c}:=\log K_{t}^{c}$.

The inclusion $Q_{t} \subset Q_{t}^{c}$ follows from convexity of the function $\check{u}_{t}^{c}(s)$ in $(s, t) \in$ $\mathbb{R}_{-}^{n} \times(0,1)$ : if $s \in Q_{t}$, then $s=(1-t) s_{0}+t s_{1}$ for some $s_{j} \in Q_{j}$, so:

$$
\check{u}_{t}(s) \leq(1-t) \check{u}_{0}\left(s_{0}\right)+t \check{u}_{1}\left(s_{1}\right)=c_{t},
$$

while we have $\breve{u}_{t}(s) \geq-c_{t}$ for all $s$. This gives us $s \in Q_{t}^{c}$.

To prove the reverse inclusion, take an arbitrary point $\xi \in \mathbb{R}_{-}^{n} \backslash Q_{t}$, then there exists $b \in \mathbb{R}_{+}^{n}$, such that

$$
\langle b, \xi\rangle>h_{Q_{t}}(b)=(1-t) h_{Q_{0}}(b)+t h_{Q_{1}}(b) .
$$

By the homogeneity of the both sides, we can assume $h_{Q_{0}}(b) \geq-c_{0}$ and $h_{Q_{1}}(b) \geq$ $-c_{1}$. Then, by (9) and (10), we have:

$$
\begin{aligned}
\check{u}_{t}(\xi) & =\sup _{a}\left[\langle a, \xi\rangle-(1-t) \max \left\{h_{Q_{0}}(a)+c_{0}, 0\right\}-t \max \left\{h_{Q_{1}}(a)+c_{1}, 0\right\}\right] \\
& \geq\langle b, \xi\rangle-(1-t) \max \left\{h_{Q_{0}}(b)+c_{0}, 0\right\}-t \max \left\{h_{Q_{1}}(b)+c_{1}, 0\right\} \\
& >(1-t)\left[h_{Q_{0}}(b)-\left(h_{Q_{0}}(b)+1\right)\right]+t\left[h_{Q_{1}}(b)-\left(h_{Q_{1}}(b)+1\right)\right]=-1,
\end{aligned}
$$

so $\xi \notin Q_{t}^{c}$.

Now, the corresponding assertions of Theorem 1 can be stated as follows.

Theorem 3 For non-pluripolar complete logarithmically convex compact Reinhardt sets $K_{0}, K_{1} \subset \mathbb{D}^{n}$, the inequality

$$
c_{t}^{n+1} \operatorname{Cap}\left(K_{t}^{\times}, \mathbb{D}^{n}\right) \leq(1-t) c_{0}^{n+1} \operatorname{Cap}\left(K_{0}, \mathbb{D}^{n}\right)+t c_{1}^{n+1} \operatorname{Cap}\left(K_{1}, \mathbb{D}^{n}\right)
$$


holds true for any $c_{0}, c_{1}>0$ and $c_{t}=(1-t) c_{0}+t c_{1}$.

In particular, the function

$$
t \mapsto\left(\operatorname{Cap}\left(K_{t}^{\times}, \mathbb{D}^{n}\right)\right)^{-\frac{1}{n+1}}
$$

is concave and consequently the function

$$
t \mapsto\left(\operatorname{Cap}\left(K_{t}^{\times}, \mathbb{D}^{n}\right)\right)^{\frac{1}{n+1}}
$$

is convex.

As we saw in the example in the previous section, sometimes one can have $u_{t}=\omega_{K_{t}^{c}}$ for $u_{j}=c_{j} \omega_{K_{j}}$, in which case (11) becomes an equality. Our next result determines when this is possible for the toric case.

Theorem 4 In the conditions of Theorem 2, the geodesic $u_{\tau}^{c}$ equals $c_{\tau} \omega_{K_{\tau}}$ for some $\tau \in(0,1)$ if and only if

$$
K_{1}^{c_{0}}=K_{0}^{c_{1}}
$$

that is, $c_{0} \log K_{1}=c_{1} \log K_{0}$.

Proof We will use the toric geodesic representation formula established in [19, Thm. 5.1]:

$$
\check{u}_{t}=\mathcal{L}\left[(1-t) \mathcal{L}\left[\check{u}_{0}\right]+t \mathcal{L}\left[\check{u}_{1}\right]\right],
$$

which is a local counterpart of Guan's result [9] for compact toric manifolds; here, $\check{u}$ is the convex image (8) of the toric plurisubharmonic function $u$.

Let $Q_{t}=\log K_{t}, 0 \leq t \leq 1$. By (9), $u_{\tau}^{c}=c_{\tau} \omega_{K_{\tau}}$ means

$$
(1-\tau) \max \left\{h_{Q_{0}}(a)+c_{0}, 0\right\}+\tau \max \left\{h_{Q_{1}}(a)+c_{1}, 0\right\}=\max \left\{h_{Q_{\tau}}(a)+c_{\tau}, 0\right\},
$$

or, which is the same,

$\max \left\{h_{(1-\tau) Q_{0}}(a)+(1-\tau) c_{0}, 0\right\}+\max \left\{h_{\tau Q_{1}}(a)+\tau c_{1}, 0\right\}=\max \left\{h_{Q_{\tau}}(a)+c_{\tau}, 0\right\}$

for all $a \in \mathbb{R}_{+}^{n}$. Therefore, $h_{Q_{0}}(a) \leq-c_{0}$ if and only if $h_{Q_{1}}(a) \leq-c_{1}$, so $c_{0} Q_{0}^{\circ}=$ $c_{1} Q_{1}^{\circ}$ and, since $(c Q)^{\circ}=c^{-1} Q^{\circ}$, we get $c_{0} Q_{1}=c_{1} Q_{0}$. Here $Q^{\circ}$ is the copolar (14) to the set $Q$, see the beginning of the next section.

\section{Covolumes}

In the toric case, the Monge-Ampère capacities with respect to the unit polydisk can be represented as volumes of certain sets [2,19]. Namely, if $K \Subset \mathbb{D}^{n}$ is complete and logarithmically convex, then $Q:=\log K \subset \mathbb{R}_{-}^{n}$ and

$$
\operatorname{Cap}\left(K, \mathbb{D}^{n}\right)=n ! \operatorname{Covol}\left(Q^{\circ}\right):=n ! \operatorname{Vol}\left(\mathbb{R}_{+}^{n} \backslash Q^{\circ}\right),
$$


where the convex set $Q^{\circ} \subset \mathbb{R}_{+}^{n}$ defined by

$$
Q^{\circ}=\left\{x \in \mathbb{R}^{n}: h_{Q}(x) \leq-1\right\}=\left\{x \in \mathbb{R}^{n}:\langle x, y\rangle \leq-1 \forall y \in Q\right\}
$$

is, in the terminology of [19], the copolar to the set $Q$. In particular:

$$
\operatorname{Cap}\left(K_{t}^{\times}, \mathbb{D}^{n}\right)=n ! \operatorname{Covol}\left(Q_{t}^{\circ}\right)
$$

for the copolar $Q_{t}^{\circ}$ of the set $Q_{t}=(1-t) Q_{0}+t Q_{1}$; we would like to stress that $Q_{t}^{\circ} \neq(1-t) Q_{0}^{\circ}+t Q_{1}^{\circ}$.

Convex complete subsets $P$ of $\mathbb{R}_{+}^{n}$ (i.e., $P+\mathbb{R}_{+}^{n} \subset P$ ) appear in singularity theory and complex analysis (see, for example, [11-13,15-17]), their covolumes (the volumes of $\mathbb{R}_{+}^{n} \backslash P$ ) being used for computation of the multiplicities of mappings, etc. Such a set $P$ generates, by the same formula (14), its copolar $P^{\circ} \subset \mathbb{R}_{-}^{n}$, whose exponential image $\operatorname{Exp} P^{\circ}$ (the closure of all points $\left(e^{s_{1}}, \ldots, e^{s_{n}}\right)$ with $s \in P^{\circ}$ ) is a complete logarithmically convex subset of $\mathbb{D}^{n}$. Since taking the copolar is an involution, the representation (13) translates coherently the inequalities on the capacities to those on the (co)volumes. Namely, $\operatorname{Cap}\left(Q_{j}\right)$ becomes replaced by $\operatorname{Covol}\left(P_{j}\right)$ with $P_{j}=$ $Q_{j}^{\circ} \subset \mathbb{R}_{+}^{n}$ for $j=0,1$, while Cap $\left(Q_{t}\right)$ has to be replaced with the covolume of the set whose copolar is $Q_{t}$, that is, with $\left((1-t) P_{0}^{\circ}+t P_{1}^{\circ}\right)^{\circ}$. The operation of copolar addition

$$
P_{0} \oplus P_{1}:=\left(P_{0}^{\circ}+P_{1}^{\circ}\right)^{\circ}
$$

was introduced in [19]. In particular, it was shown there that the copolar sum of any pair of cosimplices in $\mathbb{R}_{+}^{n}$, unlike their Minkowski sum, is still a simplex.

Corollary 1 Let $P_{0}, P_{1}$ be non-empty convex complete subsets of $\mathbb{R}_{+}^{n}$, and let the interpolating sets $P_{t}^{\oplus}$ be defined by

$$
P_{t}^{\oplus}=\left((1-t) P_{0}^{\circ}+t P_{1}^{\circ}\right)^{\circ}, \quad 0<t<1 .
$$

Then the inequality

$$
c_{t}^{n+1} \operatorname{Covol}\left(P_{t}^{\oplus}\right) \leq(1-t) c_{0}^{n+1} \operatorname{Covol}\left(P_{0}\right)+t c_{1}^{n+1} \operatorname{Covol}\left(P_{1}\right)
$$

holds true for any $c_{0}, c_{1}>0$ and $c_{t}=(1-t) c_{0}+t c_{1}$.

In particular, the function

$$
V^{\oplus}[P](t):=\left(\operatorname{Covol}\left(P_{t}^{\oplus}\right)\right)^{-\frac{1}{n+1}}
$$

is concave and, consequently, the function

$$
\rho^{\oplus}[P](t):=\left(\operatorname{Covol}\left(P_{t}^{\oplus}\right)\right)^{\frac{1}{n+1}}
$$

is convex. 
Note that the convexity of $\rho^{\oplus}$ (following from the concavity of $V^{\oplus}$ ) implies that the function

$$
\tilde{\rho}^{\oplus}[P](t):=\left(\operatorname{Covol}\left(P_{t}^{\oplus}\right)\right)^{\frac{1}{n}}
$$

is convex as well. Since $\tilde{\rho}^{\oplus}$ is a homogeneous function of $P$, that is,

$$
\tilde{\rho}^{\oplus}[c P](t)=c \tilde{\rho}^{\oplus}[P](t)
$$

for all $c>0$ and $0<t<1$, its convexity is equivalent to the logarithmic convexity of the covolumes, established in [8] by convex geometry methods:

$$
\operatorname{Covol}\left(P_{t}^{\oplus}\right) \leq \operatorname{Covol}\left(P_{0}\right)^{1-t} \operatorname{Covol}\left(P_{1}\right)^{t},
$$

which is just another form of the Brunn-Minkowski type inequality (4). Therefore, the concavity of the function $V^{\oplus}$ is a stronger property than just the logarithmic convexity of the covolumes.

Acknowledgements The author would like to thank the anonymous referee for suggestions that have improved the presentation.

Funding Open access funding provided by University Of Stavanger.

Open Access This article is licensed under a Creative Commons Attribution 4.0 International License, which permits use, sharing, adaptation, distribution and reproduction in any medium or format, as long as you give appropriate credit to the original author(s) and the source, provide a link to the Creative Commons licence, and indicate if changes were made. The images or other third party material in this article are included in the article's Creative Commons licence, unless indicated otherwise in a credit line to the material. If material is not included in the article's Creative Commons licence and your intended use is not permitted by statutory regulation or exceeds the permitted use, you will need to obtain permission directly from the copyright holder. To view a copy of this licence, visit http://creativecommons.org/licenses/by/4.0/.

\section{References}

1. Abja, S.: Geometry and topology of the space of plurisubharmonic functions. J. Geom. Anal. 29(1), 510-541 (2019)

2. Aytuna, A., Rashkovskii, A., Zahariuta, V.: Widths asymptotics for a pair of Reinhardt domains. Ann. Polon. Math. 78, 31-38 (2002)

3. Bergh, J., Löfström, J.: Interpolation Spaces. An Introduction. Springer, Berlin (1976)

4. Berman, R.J., Berndtsson, B.: Moser-Trudinger type inequalities for complex Monge-Ampère operators and Aubin's "hypothèse fondamentale". arXiv:1109.1263

5. Calderón, A.-P.: Intermediate spaces and interpolation, the complex method. Studia Math. 24, 113-190 (1964)

6. Cordero-Erausquin, D.: Santaló's inequality on $\mathbb{C}^{n}$ by complex interpolation. C. R. Acad. Sci. Paris Ser. I 334, 767-772 (2002)

7. Cordero-Erausquin, D., Klartag, B.: Interpolations, convexity and geometric inequalities. In: Geometric Aspects of Functional Analysis, Lecture Notes in Math., vol. 2050, pp. 151-168. Springer, Berlin (2012)

8. Cordero-Erausquin, D., Rashkovskii, A.: Plurisubharmonic geodesics and interpolating sets. Arch. Math. (Basel) 113(1), 63-72 (2019)

9. Guan, D.: On modified Mabuchi functional and Mabuchi moduli space of Kähler metrics on toric bundles. Math. Res. Lett. 6(5-6), 547-555 (1999) 
10. Guedj, V. (ed.): Complex Monge-Ampère Equations and Geodesics in the Space of Kähler Metrics. Lecture Notes in Math., vol. 2038. Springer, Berlin (2012)

11. Kaveh, K., Khovanskii, A.: Convex bodies and multiplicities of ideals. Proc. Steklov Inst. Math. 286(1), 268-284 (2014)

12. Khovanskil, A., Timorin, V.: On the theory of coconvex bodies. Discrete Comput. Geom. 52(4), 806823 (2014)

13. Kim, D., Rashkovskii, A.: Higher Lelong numbers and convex geometry. To appear in J. Geom. Anal.; arXiv: 1803.07948

14. Klimek, M.: Pluripotential Theory. Oxford University Press, London (1991)

15. Kouchnirenko, A.G.: Polyèdres de Newton et nombres de Milnor. Invent. Math. 32, 1-31 (1976)

16. Rashkovskii, A.: Newton numbers and residual measures of plurisubharmonic functions. Ann. Polon. Math. 75(3), 213-231 (2000)

17. Rashkovskii, A.: Tropical analysis of plurisubharmonic singularities. In: Tropical and Idempotent Mathematics, Contemp. Math., vol. 495, pp. 305-315. Amer. Math. Soc., Providence (2009)

18. Rashkovskii, A.: Local geodesics for plurisubharmonic functions. Math. Z 287, 73-83 (2017)

19. Rashkovskii, A.: Copolar convexity. Ann. Polon. Math. 120(1), 83-95 (2017)

Publisher's Note Springer Nature remains neutral with regard to jurisdictional claims in published maps and institutional affiliations. 